\title{
A Novel Dual Traffic/Flash Flood Monitoring System Using Passive Infrared/Ultrasonic Sensors
}

\author{
Mustafa Mousa*, Enas Oudat* and Christian Claudel ${ }^{\Psi}$ \\ *King Abdullah University of Science and Technology \\ $\Psi$ The University of Texas at Austin
}

\begin{abstract}
Floods are the most common type of natural disaster, causing thousands of casualties every year. Among these events, urban flash floods are particularly deadly because of the short timescales on which they occur, and because of the high concentration of population in cities. Since most flash flood casualties are caused by a lack of information, it is critical to generate accurate and detailed warnings of flash floods. However, deploying an infrastructure that solely monitor flash floods makes little economic sense, since the average periodicity of catastrophic flash floods exceeds the lifetime of a typical sensor network.

To address this issue, we propose a new sensing device that can simultaneously monitor urban flash floods and another phenomenon of interest (traffic congestion on the present case). This sensing device is based on the combination of an ultrasonic rangefinder with one or multiple remote temperature sensors. We show an implementation of this device, and illustrate its performance in both traffic flow and flash flood sensing. Field data shows that the sensor can detect vehicles with a $99 \%$ accuracy, in addition to estimating their speed and classifying them in function of their length. The same sensor can also monitor urban water levels with an accuracy of less than $2 \mathrm{~cm}$. Two of the sensors have been deployed in a flood prone area, where they captured the only (minor) flash flood that occurred over the one-year test period, with no false detection, and an agreement in the estimated water level estimate (during the flash flood event) of about $2 \mathrm{~cm}$.
\end{abstract}

Keywords-Flood detection, Speed estimation, Vehicle classification, Artificial Neural Networks, Wireless Sensor Networks.

\section{INTRODUCTION}

Wireless sensor networks (WSNs) are widely used for monitoring and control applications such as environmental surveillance [15] or industrial sensing [19], [14], or in the present case flash flood detection. Floods are one of the most commonly occurring natural disasters [26], accounting for more than half of natural disasters worldwide. Floods caused more than 120,000 fatalities in the world between 1991 and 2005 [1], and are a major problem in many areas of the world. Recent examples of catastrophic flood events include the 2014 floods in Kashmir, India (between 300 and 400 casualties), the 2013 flash floods in Argentina (more than 75 casualties) or the 2012 flash floods floods in Kyushu, Japan (32 casualties).

Among floods, flash floods are short fuse weather events that usually peak in less than six hours. Most flood fatalities are caused by flash floods, and most flash flood victims die due to drowning [24], [9], which could be avoided by providing accurate flash flood maps to the population in real time (through standard information channels). Unfortunately, at the present time little warning exists beyond weather forecasts, which are nonspecific and unreliable, i.e. these warnings are associated with a very high false alarm rate.

Monitoring floods in real time somehow requires sensing the flooding conditions [27], which includes water presence, water levels, water velocity and rain rate. Fixed water level sensors are only adapted to river monitoring and are not adapted to situations in which the runoff does not correspond to permanent rivers. Instrumenting entire hydrological basins, which can cover hundreds of square miles, is economically infeasible, since the lifetime of a sensor network is usually less than the mean time between catastrophic flood events. Satellites are also unable to accurately monitor water levels and water flows remotely: optical measurements are impossible during floods due to the severe rains associated with floods, and the vertical resolution of currently available synthetic aperture radars is insufficient.

For all these reasons, a wireless sensor network would be the best approach to flash flood monitoring in cities. To be economically viable, this flash flood sensor network must have a secondary sensing capability that justifies the cost of its purchase and deployment. Other critical issues in cities include for instance air pollution or traffic congestion monitoring. Intelligent Transportation Systems (ITS) are critical in reducing traffic congestion, increasing safety and productivity. Since traffic congestion is a worsening issue in most countries, monitoring congestion levels in real time is of critical importance: it not only enables better information to be relayed to users, but also paves the way to efficient traffic control system [28]. Traffic monitoring includes the measurement of vehicle speeds, flows, density and the classification of vehicles in different categories (for instance buses, trucks or light vehicles). Current ITS systems typically rely on wired sensor networks, for instance the PeMS system in California [21], which do not have specific power requirement constraints. Alternate systems do exist, such as wireless sensor networks [12], which are much easier and cheaper to install and operate.

We presently focus on sensors that could monitor simultaneously flash floods and traffic congestion in cities. Multiple sensing principles can be thought of in the context of traffic monitoring, including magnetometers [13], traffic cameras [31], acoustic sensors [17], [16], RADAR (Radio Detection And Ranging) or LIDAR (Laser Infrared Detection And Ranging) [5]. These sensing methods are typically expensive, since they require advanced processing circuitry, specially in the case of cameras, RADARs and LIDARs. In contrast, passive 
infrared sensors (PIRs), [4], [36] and ultrasonic rangefinders [23] are low cost, reliable and small, and can be readily used for both sensing applications. They are particularly suitable for traffic monitoring systems as they can be easily deployed on the road sides or on traffic lights, and can measure without contact with the target. They can also monitor distances, which make them particularly suitable for the present flash flood monitoring application. In this article, we propose a new type of traffic and flash flood sensors based on the combination of ultrasonic rangefinders with one or multiple passive infrared temperature sensors. This sensor combination can be used as a backbone for a dual urban traffic/flash flood wireless sensor network, since it can monitor vehicle speeds, counts, density and vehicle types as well as pluviometry, water presence and water level with a relatively high accuracy.

The rest of this article is organized as follows. Section II summarizes the previous work in the context of traffic and flash flood monitoring. Section III describes the dual ultrasonic/passive infrared sensor network used in this study, and the associated traffic flow and flash flood measurement principle by introducing an implementation of the traffic and flash flood sensor, as well as a custom-designed processing board. Section IV describes a machine-learning framework for estimating the level of water in an urban environment using a combination of ultrasonic distance measurement and air/ground temperature measurements. In section VI, we show that the same sensor can accurately monitor traffic parameters (flow, density, velocity and vehicle classes) by using a Bayesian network fed by the wavelet transforms of the ground temperature measurements.

Finally, we show the performance of the system in both flash flood and traffic monitoring in sections $\mathrm{V}$ (giving a real flood incident in V-E) and in VI respectively.

\section{PREVIOUS WORK}

Flash flood events are catastrophic flooding events caused by high rainfall events occurring over short periods of time. Current flash flood estimation algorithms rely on forward simulations of the associated hydrological basin using hydrological models, which are combined with real-time measurements (or prior informations) using for example Ensemble Kalman Filtering [34] or Particle Filtering. The underlying models are typically the Shallow Water Equations (SWE), which are an approximation of the Navier Stokes equations for problems involving shallow water levels (which typically occur in floods). The shallow water equations are a partial differential equation (PDE) that describe the evolution of a body of water under the influence of gravity, ground friction, and rains. The state of the body of water is encoded by two functions, the velocity function and the water level function. In almost all instances of flash floods, the diffusive wave approximation to the shallow water equations (DSW, [6]) can be used. The DSW equations are a simplification of the shallow water equations, used to model flows where the vertical momentum is small relative to the horizontal. With this approximation a single function (for instance the level of water) is sufficient to describe the state of the system.
The main issue arising when dealing with hydrological models is their inaccuracy, particularly because of large uncertainties on the runoff coefficients or the terrain altitudes. As an example, parameters of the inundation models such as the Manning coefficient are highly uncertain, even with high resolution satellite maps or LIDAR surveys [33]. For instance, the Manning coefficient associated with boulder terrain can vary from 0.04 to 0.07 , a $70 \%$ relative uncertainty [29].

An example of river level estimation using the combination of hydrological models with rain rate data is illustrated in article [3] by $\mathrm{Li}$ et al, for monitoring and predicting floods in Africa. In this article, discharge rates of rivers are estimated using satellite precipitation data as well as ground precipitation data from a network of weather stations. Despite the very high accuracy of satellite precipitation data (with a spatial resolution on the order of a few hundred meters), the actual discharge rates in the rivers vary significantly from the results of the simulations. Furthermore, a relatively high proportion of floods occur in desert areas (for instance the 2014 Indian/Pakistan flash floods), and are not associated with rivers that exist for long periods of time, and for which no extensive satellite data allows the calibration of the model.

Recent efforts have shifted towards adding real time flooding data (for instance in the form of water level or water velocity measurements) to the estimation process, to improve the quality of flood estimates over pure simulation. One such flood WSN is described in Basha et al [7], for monitoring and predicting water levels in an hydrological basin located in Nicaragua, using direct water-level measurements as well as precipitation data.

Other flood monitoring systems exist, in particular satellitebased or UAV-based (unmanned-air-vehicle) optical systems. For instance, an UAV-based system has been developed by [32]. More recently, in 2011, a Predator B drone from the US Customs and Border Protection office was used for flood mapping in Minnesota and North Dakota. However, in all of these operations, the focus of the operations was flood damage assessment, not real-time flood monitoring. All UAVs used in this context mapped the area using cameras, which cannot be applied to most flash flood sensing applications in which visibility is typically very low (high rains, mist, sand).

The use of synthetic aperture radars (SARs) in lieu of cameras is also discussed in [32]. While SARs are impervious to visibility problems, and can map extensive areas even during floods, their resolution is far too low for real-time monitoring [32], and are thus only used for post damage assessment. Typical SARs resolution is between 1 to $20 \mathrm{~m}$, though only 6 inches of fast moving water can knock an adult over [2]. An additional issue with SARs is their sensitivity to turbulence [18], which makes them impractical for flash floods, which are usually caused by thunderstorm cells.

\section{SENSING PRINCIPLE}

\section{A. Sensor design considerations}

In this article, our objective is to build a sensor system that can simultaneously monitor one parameter associated with flash floods (for instance water level or water velocity, 
following the DSW equations formulation), in addition to performing traffic sensing measurements, using the minimal amount of components, and for the lowest possible cost and the highest possible accuracy.

The commonality between both applications leaves two possible choices for the sensing: contact sensors (for instance pressure sensors that could both sense water level and vehicle presence, and non-contact sensors measuring physical parameters associated with both flash floods and traffic). Sensor systems that only work for specific applications (for instance water velocity sensors) are excluded from this analysis since they do not allow the monitoring of both phenomena.

Sensors have been investigated in the past for flood monitoring applications, in particular ultrasonic water level measurements on bridges [30], [11] or pressure sensors for water level measurements of rivers [7]. In terms of contact sensors, pressure sensors are the only sensors that could be affected both by traffic flow and flash floods. They could be designed as pressure transducers (or pressure transducer arrays). Pneumatic tubes, piezoelectric cables or strain gauges have long been used for traffic flow monitoring, for instance for weigh-in-motion [37] applications. There are two main issues associated with contact sensors that make them impractical for the proposed application:

- Pressure readings are simultaneously affected by water velocity (dynamic pressure) and water level (static pressure). The sensor readings would not allow one to differentiate between a low, fast moving stream of water and a high level of standing water, and would be sensitive to the orientation of the sensor surface with respect to the stream of water. The inversion from pressure measurements require additional data (for instance velocity obtained from a video camera).

- The water during flash flood events can carry a large amount of debris and sediments, which may cause a pressure sensor to fail.

Another issue is the impossibility for an operator to ascertain that the sensor is still functional at all times: indeed, the system would have to be periodically checked by exerting ground pressures on the order of $1 \mathrm{kPa}$ over the sensor to check its response, which adds to the operational cost.

In the present case, the constraints detailed above prevent contact sensors such as pressure transducers from being used. Among non-contact sensing technologies, multiple technologies could be thought of, including cameras, ultrasonic rangefinders and Ultra Wide Band (UWB) radars. Other noncontact traffic sensors do exist, for instance inductive loops [25] or magnetic sensors [12], though these sensors would be unable to evaluate the level of water from inductance measurements. Indeed, the relative magnetic permeability of water is extremely close to the relative magnetic permeability of air (0.999992 vs 1.00000037$)$, and the magnetic permeability of flood water (with unknown concentrations of sediment and debris) cannot be known in advance.

Cameras could theoretically be used to simultaneously monitor traffic and flash floods. They have been extensively used in traffic monitoring applications [10]. In terms of flash flood sensing, their main limitation is related to the low visibility occurring during flash flood events, and the tracking of water velocity (which is different from the velocity of surface waves).

Non contact level measurement systems include sound waves based systems (ultrasonic rangefinders) and electromagnetic waves based systems (for instance LIDARs, or Ultra Wide Band radars). LIDARs have the required theoretical accuracy, but have several drawbacks which make them impractical. Indeed, their narrow field of view (typically a few degrees) makes the water level measurements very sensitive to the surface waves, lowering the accuracy. In addition, water tends to absorb infrared radiation, which results in a weaker return signal, further reducing the measurement accuracy. LIDARs also consume much more (about $500 \mathrm{~mW}$ in continuous operation) than other ranging devices such as ultrasonic rangefinders or UWB radars, which is an issue for non grid-powered sensor networks.

UWB radars can be used to monitor distances (and simultaneously sense traffic conditions), but commercial UWB systems have typically a low accuracy, of tens of inches, which make them impractical for flash flood monitoring.

In contrast, ultrasonic rangefinders are relatively inexpensive and accurate. They have been used extensively for traffic flow monitoring, for instance in North America [35]. Mounted in overhead configuration, they can also theoretically monitor the distance between the sensor, and are much less sensitive to perturbations caused by surface waves than LIDARs, since their field of view is on the order of tens of degrees. Water also causes strong signal reflections, which are easily picked up by the sensor, unlike infrared radiation used in LIDARs.

For all the reasons above, we investigate in this article a sensor system based on an ultrasonic rangefinder. Such sensors are typically low power $(<10 \mathrm{~mW}$ in sensing mode $)$, low cost, weather resistant, and high accuracy $(0.5$ in for the system used in this article, though more recent systems are now capable of $\mathrm{mm}$ resolution). This sensor is also capable of selfmonitoring, since it constantly measures the level of water: unlike contact-based systems (such as pressure sensors) the infrastructure operator can know in real time if a subsystem is malfunctioning.

Unlike electromagnetic waves, the velocity of ultrasound (or sound) waves depends on the environmental condition, the main parameter affecting the speed of sound being temperature. Since the temperature of the air layer located between the ultrasonic sensor and the ground is highly non uniform, we need a sensor simultaneously measuring the temperature at the level of the sensor and the temperature at the ground level. Modern infrared thermopiles can perform both measurements simultaneously.

\section{B. Sensor node}

The traffic and flash flood sensor node that we investigate in this article contains six passive infrared sensors and one ultrasonic rangefinder. The presence of multiple passive infrared sensors allows traffic monitoring on a higher number of traffic lanes. The six passive infrared thermopiles are Melexis MLX90614 connected to the platform via SMBus. These sensors simultaneously measure the temperature in their field of view 


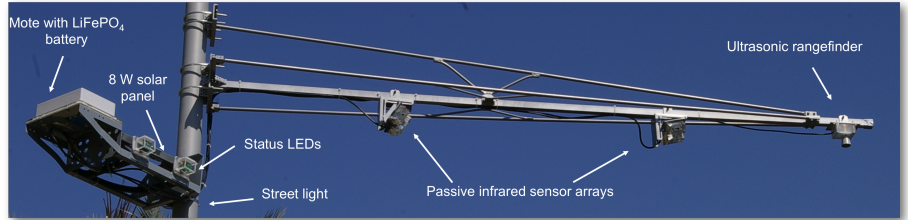

Figure 1. Traffic/Flood sensor node installed on a public street light.

and their own temperature. The ultrasonic rangefinder is a MaxBotix MB7066 measuring its distance to below objects. This sensor is connected to the microcontroller via a serial port. In the tested configuration the ultrasonic rangefinder generates data at a $10 \mathrm{~Hz}$ rate, and the measurement frequency of each of the six thermopiles is set at $16 \mathrm{~Hz}$. The measurements are sent wirelessly to a sink node, and are then pushed to an input database for processing.

To mount the sensor in an overhead configuration, we designed, using CAD software, a lightweight aluminum alloy frame that weights less than $6 \mathrm{~kg}$, and can be mounted on regular street lights, extending 3 meters away from the curb side.

Four sensors have been deployed to date, including two sensors in a flood prone area. An example sensor is shown in Figure 1.

\section{Microcontroller unit}

The microcontroller platform used in this article has been developed (by our group [22]) for this specific sensing application around a $168 \mathrm{MHz}$ ARM Cortex M4 (STM32F 407 from ST Microelectronics), with $192 \mathrm{kB}$ of RAM. The platform is illustrated in Figure 2. We selected this specific microcontroller for the application since it satisfies the requirements of our estimation problem and best balances the tradeoff between RAM, power consumption, computation and cost. This sensing platform has multiple peripherals, including a micro-SD card reader, a flash memory module, and a complete energy monitoring circuitry for battery level estimation and solar power generation estimation. This energy monitoring subsystem is very valuable in practice since the sensor system is solar powered (to minimize deployment costs), and uses a Lithium Iron Phosphate battery for energy storage. While very reliable under a variety of environmental conditions, Lithium Iron Phosphate batteries tend to have a very flat discharge curve, and the remaining battery level cannot be reliably estimated using voltage measurements alone.

In addition to executing the computational tasks related to sensing (i.e. processing the raw data generated by the sensors into traffic flow and flash flood measurements), the microcontroller platform executes network routing tasks and energy monitoring tasks

\section{FLASH FLOOD MONITORING}

\section{A. Flood detection problem definition}

We deployed a prototype sensor in November 2013 to evaluate the need of advanced algorithms for estimating the

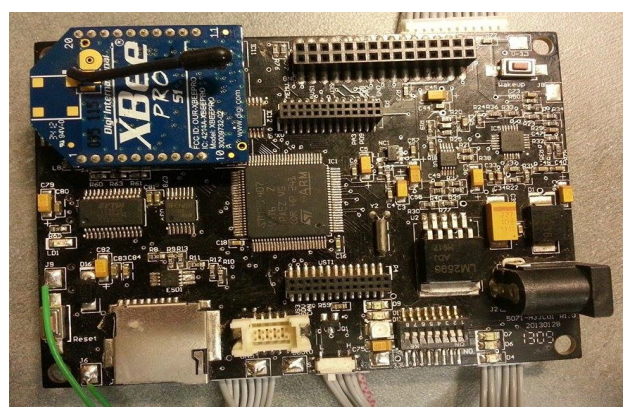

Figure 2. Custom-developed 32-bit microcontroller platform (4in x 3in) with connected XBee module. The microcontroller platform includes a XBee radio, a solar power charging and monitoring circuit and a SD card for data storage. It is powered by a $10 \mathrm{~W}$ solar panel, and energy is stored in a single cell Lithium-Iron-Phosphate battery of capacity 8A.h.

level of water using the raw ultrasonic distance and air/ground temperature measurements.

The temperature and distance to the ground measurements generated by the sensor vary significantly over the period (about $12 \mathrm{~cm}$ ) despite the fact that no flooding occurred over this period (tested under normal conditions so that sensor fluctuations are only due temperature change). These variations in observed distance are caused by the dependency of the speed of sound upon air temperature. For typical measurements, the air temperature effects are usually negligible, specially when the temperature in the air layer can be approximated by a single value. For our sensor, installed at a height of 5 meters (to clear the below traffic) and at usual temperatures, a $1^{\circ} \mathrm{C}$ increase in average temperature causes a $1 \mathrm{~cm}$ increase in the measured distance.

The problem of estimating the true distance between the sensor and the ground from our available measurements can be formulated as follows. The ultrasonic rangefinder actually measures a time-of-flight (which is converted into an estimated distance by the electronics of the sensor, assuming a fixed speed of sound). The time of flight $T(t)$ of the ultrasonic wave (measured at a given time $t$ ) can be computed as follows:

$$
T(t)=2 \int_{0}^{z_{0}} \frac{d z}{c(\theta(z, t))}
$$

where $\theta(z)$ is the air temperature at altitude $z$. One of the main difficulties arising in this problem is the estimation of this integral, since the function $\theta(\cdot)$ depends on multiple factors that are not directly measured by the sensor, including for instance cloud coverage, presence of shadows from buildings, heat island effects. Theoretically, $\theta(\cdot)$ could be computed as the solution to the heat equation. In practice, it is infeasible to model the air layer temperature using a PDE, since the boundary conditions of the problem are unknown (for example: solar forcing, ground albedo, presence of wind). We thus investigate various non-PDE-based models to perform this temperature compensation. 


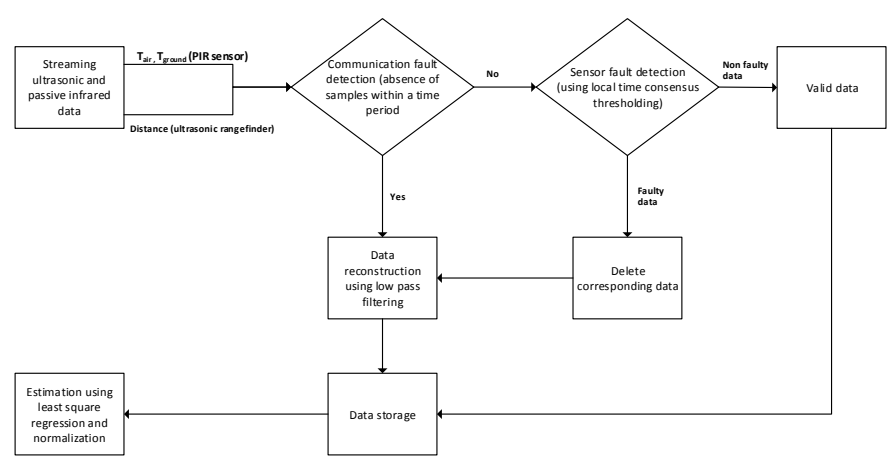

Figure 3. This figure illustrates the preprocessing of the passive infrared data. The processed data is an input to the supervised learning scheme.

\section{B. Preprocessing of temperature measurements}

The raw data generated by the infrared temperature sensors and the ultrasound rangefinder have different scales, and are sometimes exhibiting inconsistencies.

We traced the source of these inconsistencies to an incorrect (deviating from the manufacturer specifications) behavior of the charging circuitry, which randomly resets in some narrow, low input current range (occurring mainly during sunrise and sunset). In addition, missing data can be caused by network faults, which in practice were mainly related to the failure of the computer connected to the sink node. To address these issues, we designed a preprocessing filter that identifies anomalies in the data (from the infrared temperature sensors and the ultrasound rangefinder), and remove these anomalous data using local time consensus thresholding. The missing data is reconstructed using a least squares regression technique. This approximation is valid since usually only a few data points are affected by the incorrect behavior of the charging circuitry.

Precisely, faulty data (caused by the non-nominal behavior of the charging circuitry) is detected by analyzing the consensus among all six infrared temperature sensors in the node. We design a sliding window of one day in which we calculate the mean $\mu$ and the standard deviation $\sigma$ of the passive infrared sensors. The records that deviate more than $3 \sigma$ from the mean are considered anomalies, and excluded from the dataset. Since the main cause of sensor noise is thermal noise, the sensor measurements follow a normal distribution. Hence, $99.7 \%$ of the values fall within the $[\mu-3 \sigma, \mu+3 \sigma]$ window. Most of the data excluded using this approach is indeed faulty data. A general least square regression model is then used to approximate the missing values between points.

The network faults are identified by detecting time periods that have more than 15 minutes (i.e. 90 samples at the $100 \mathrm{mHz}$ message update rate) with no activity. No data reconstruction is attempted in these situations.

The flow chart for the preprocessing stage is illustrated in Figure 3.

An illustration of the results of the preprocessing scheme is shown in Figure 4

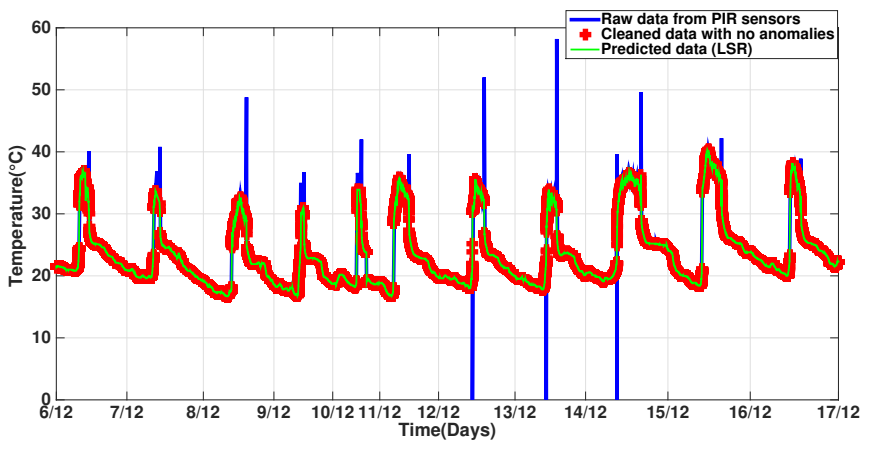

Figure 4. Comparison between raw PIR sensor data (blue) and reconstructed PIR data (green) at the end of the preprocessing stage.

\section{Naive temperature correction}

We first investigate simple temperature correction schemes using direct (air and ground) temperature measurements from the sensors [8]. The measured time of flight is given by equation 1 in which $\theta(z, t)$ is unknown, though the integral itself may be approximated by a simple direct relation with temperature measurements. Let us first assume that $\theta(\cdot, t)$ is constant, that is, the temperature in the air column is spatially uniform.

In Figure 5, we assume that the air temperature in the column is identical to the ambient temperature. The ambient temperature is either obtained from the thermopile sensor placed on the sensor itself, or from air temperature measured at the nearby weather station.
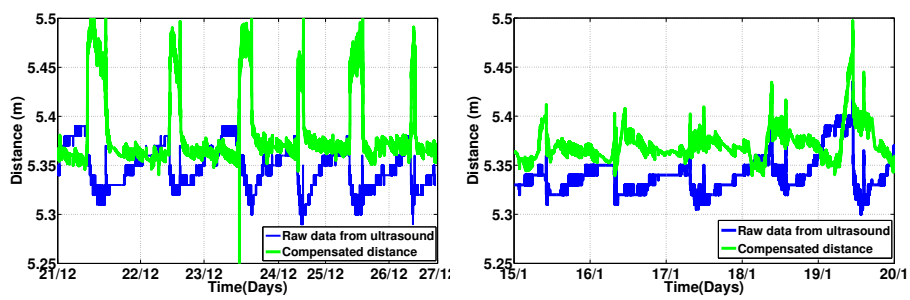

Figure 5. Predicted distance measurement computed using ambient temperature sensor measurements (left), and weather station air temperature measurements (right). As can be seen from this Figure, assuming that the air temperature is uniform and equal to the ambient temperature measurements from the sensor does not yield sufficiently accurate results, with errors reaching up to $15 \mathrm{~cm}$. As expected, the distance estimation during the night (no solar forcing) is comparably very good, with less than $1 \mathrm{~cm}$ error in average.

As can be seen from Figure 5, the estimation during the night is very good, though the compensation fails during the day. This is caused both by the assumption that the air temperature is uniform, and by the assumption that the ambient temperature measured by the sensor is a good proxy of the air temperature. Since the sensor is heated by the sun, its temperature is usually higher than the air temperature.

Since the ambient temperature measured by the PIR sensor is higher than the air temperature, we use standard air temperature measurements from a nearby weather station as a proxy for the average temperature in the air column. True 
air temperature measurements from the nearby weather station do not significantly improve the results. This shows that the assumption of a constant temperature in the air column is wrong.

To model this effect, we can for instance assume that the temperature is a linear function of the altitude, between the ground and the sensor, as follows:

$$
T(h)=T_{g}(t)+\frac{T_{a}(t)-T_{g}(t)}{L} h
$$

where $L$ represents the fixed distance between the sensor node and the ground, and $h$ represents the elevation above the ground. Unfortunately, this assumption does not significantly improve the results with respect to the two previous assumptions. The main issue is that a linear dependency between temperature and height does not take into account the effects of the wind or of urban cast shadows, which are unmeasured variables of the problem.

This preliminary analysis shows that more advanced methods are needed to estimate the distance between the sensor and the ground accurately, in function of the current and past measurements of temperature.

\section{Temperature compensation using supervised learning}

Supervised learning has been successfully applied to a wide range of applications. Supervised learning algorithms model the functional relationship between inputs and outputs, and do not require any knowledge of the internal dynamics of the system.

In the present study, the complex relationship between environmental parameters and temperature correction can be modeled through supervised learning: the input correspond to the ambient and ground temperatures, and the output corresponds to the actual distance between the sensor and the ground. Note that when floods do not occur (i.e. most of the time), the actual distance between the sensor and the ground is perfectly known.

\section{Flash Flood Detection Performance}

This section describes the performance of different models in terms of water level estimation.

\section{A. Linear ARMAX model}

Autoregressive Moving Average models can be fitted using standard system identification techniques. Being a linear model, the estimation of the ARMAX parameters boil down to linear least squares, which can easily be done onboard a wireless sensor node.

\section{B. Nonlinear supervised learning models}

In all subsequent comparisons we consider models that have an identical number of parameters. The results show that non-linear supervised machine learning approach significantly outperforms an ARMAX model. The relative performance of the prediction of both models, on the validation dataset, is shown in Figure 6.

Our data shows that an absolute accuracy (in the norm infinity sense) of two centimeters can also be reached with supervised learning using nonlinear regression functions. Choosing a good nonlinear dynamical model is complex, however, since the data exhibits multiple time scales (slow variations of the weather at the scale of days, fast variations of the sun irradiance at the scale of hours, caused by clouds, and very fast temperature changes caused by rains or hot air currents) that are difficult to model.

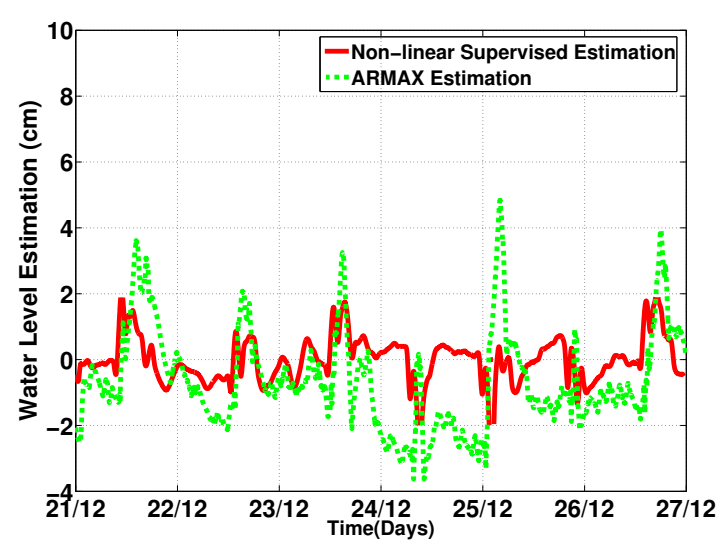

Figure 6. Estimated water level on a 7-days validation dataset (including rain events but no floods) using non-linear supervised learning or ARMAX.

\section{Artificial Neural Network based supervised learning}

As mentioned earlier, supervised learning is particularly adapted to the present problem, since the true distance between the sensor and the ground (and thus the true value of the correction factor to apply) is known most of the time. Among all possible statistical learning techniques, we choose to use ANNs, for their favorable convergence properties. Artificial neural networks are well suited to many problems due to their "universal approximation" property: any continuous function can be uniformly approximated to an arbitrary level of accuracy by neural networks, given enough hidden units. for a variety of continuous nonlinear hidden-layer activation functions [20].

We tested the neural network model using the neural network toolbox of Matlab, running on a Mackbook i7. We chose to train the neural network using the LevenbergMarquardt algorithm with one hidden layer of 10 neurons in a two inputs/one output setting. In performance tests the root mean square error (RMSE) of the estimates obtained through ANN only improve marginally as we increase the number of neurons past 5 neurons.

The dataset consists of 205000 data points, collected over a six-month period. We arbitrarily divide it into a training dataset, a validation dataset and a testing dataset. The training dataset corresponds to $70 \%$ of the overall data, while the validation and testing datasets each correspond to $15 \%$ of the data. 
Figure 7 shows the histogram of the error between output and targets for the training, validation and testing samples.

The absolute difference between predicted and actual distance measurements is shown in Figure 7. Since no flood occurred during this period, the actual water level was always $0 \mathrm{~cm}$. As can be seen from the figure, the error is less than 1.85 $\mathrm{cm}$. This low prediction error shows the good performance of a neural network on water level prediction.
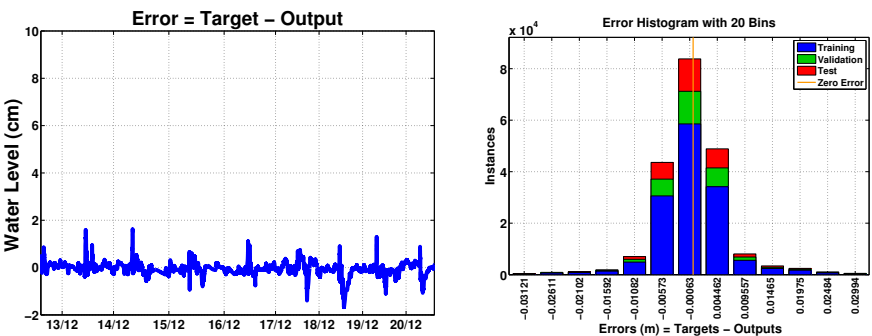

Figure 7. Left: Water level prediction during a week of December 2013 Since no flood occurred during this period, the actual water level was always $0 \mathrm{~cm}$. The deviation of predicted value from 0 can be considered as prediction error, which is less than $1.85 \mathrm{~cm}$. Right: error distribution over the training, testing and validation datasets.

\section{Comparison of prediction models}

Table I compares various estimation techniques that we investigated for the present application. Their performance is compared in terms of prediction accuracy (measured by the maximum absolute error $\left(L_{\infty}\right)$ and RMSE), efficiency (computational time in both training and prediction) and prediction stability. In terms of accuracy, the two machine learning methods perform better than both naive compensation and linear ARMAX models. The best performance is given by the ANN supervised learning algorithm. With respect to computational time, the naive compensation is associated with a zero training time, since it is memoryless. The training time required by the ARMAX estimation method is almost negligible, since it involves linear least squares. Each of the ML methods involves a more lengthy training process, and thus require more time for learning. However, for a practical sensor network the most important consideration is the computational time required by the prediction step, since the training process can be conducted off-site, using regular computers. In any of the tested algorithms, the estimation of 10,000 data points takes less than a second on a Mackbooki 7 computer. Using standard Dhrystone performance indices, we evaluated the sensor platform to be about 100 times slower than the Mackbooki7, which still ensures that a single data point can be computed within $10 \mathrm{~ms}$, considerably faster than the update rate of the sensor $(10 \mathrm{~s})$.

Table I. COMPARISON OF THE ESTIMATION PERFORMANCE OF FOUR DIFFERENT MODELS

\begin{tabular}{|l|l|l|l|l|l|}
\hline Model & $L_{\infty}(\mathrm{cm})$ & RMSE & \multicolumn{2}{|l|}{ Computational time (sec.) } & \multirow{2}{*}{ Error Std. } \\
\cline { 4 - 5 } & & & Training & Prediction & \\
\hline Naive Compensation & 15.3 & 0.0547 & 0 & 5.2727 & 0.0504 \\
\hline ARMAX & 4.9 & 0.0164 & 0 & 272.2731 & 0.0152 \\
\hline NL Regression (ML) & 2.57 & 0.0082 & 28 & 5.6629 & 0.0083 \\
\hline NN (ML) & 0.6 & 0.0058 & 975 & 5.2231 & 0.006 \\
\hline
\end{tabular}

\section{E. Validation on an actual flooding incident}

Flood incidents are extremely rare. We however validated the performance of the sensor over a single actual flooding incident, which has been the only flooding incident occurring in the area covered by the four deployed sensors to date. A minor flooding incident occurred on the Campus of Umm Al Qura University, in the city of Mecca, Saudi Arabia, in which two of the sensors ("8F48" and "D3CB", from the last four digits of their MAC address) are deployed. The flood occured on May 9, 2014. Witnesses in the area where the flood occurred estimated the water level to be less than $10 \mathrm{~cm}$, though they could not measure it precisely, since the flooding event started at 11 PM local time.

This incident has been independently captured by the two sensors, which are located 10 meters apart. The water level estimates are illustrated in Figure 8 for both sensors. As can be seen from this Figure, the onset of the flood is clearly detected, and corresponds to a significant rise in the water level estimate. We can also see the time of onset of the flood is identical for both sensors, and that the estimated water levels are of the same order of magnitude (7 to $9 \mathrm{~cm}$ ).

During this flooding event, the sink node failed shortly after the flood occurred, around 11:30 PM local time, due to electrical grid shutdown related to the flooding event. Nevertheless, the sensors have demonstrated that they could capture this event. No false positive (using a $2 \mathrm{~cm}$ threshold) has occurred since the deployment of the four sensors.
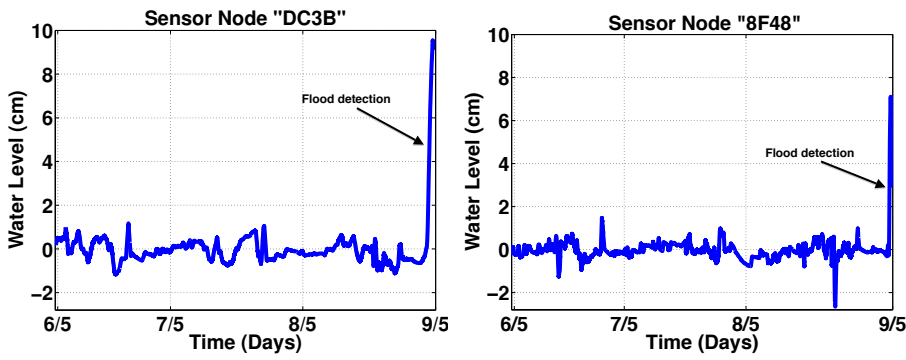

Figure 8. Water level estimation of the validation dataset on sensor nodes "DC3B" and "8F48" during the sole flooding event occurring over the testing period.

\section{TRAFFIC MONITORING PERFORMANCE}

Due to the environmental effects on the detection by passive infrared sensors, measurements have been collected in different times and weather conditions which is important to have a robust detection and speed estimation methods.

\section{A. Sensing principle}

Passing vehicles are detected by the passive infrared sensors from the thermal perturbations that they create. The rangefinder can also detect the presence of vehicles below it from the distance measurement timeseries. In the present article, we focus on temperature-based sensing, since the detection of vehicles by the ultrasonic rangefinder is rather straightforward, and can be done using thresholding only. 


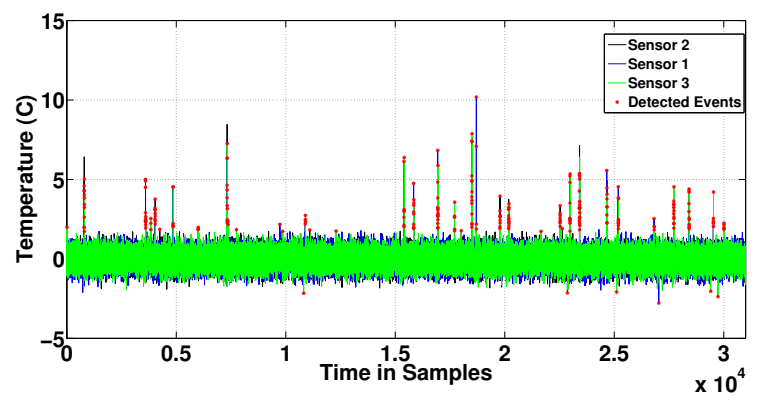

Figure 9. Time series and the detected events by all sensors

\section{B. Vehicle detection using Gaussian Mixture Models}

The detection of vehicles using passive infrared temperature measurements is presently done using Gaussian Mixture models (GMM), in which we classify the data into events, an event corresponding to a passing vehicle. Gaussian Mixture models (GMM) are probabilistic models that assume the existence of sub-populations within the overall population in the observed data. They are used to make statistical inference about the properties of these sub-population given the observed data. Based on this approach, sensor measurements, $Y^{k}$, are assumed to be a mixture of two Gaussian distributions corresponding to event: $\operatorname{Pr}\left(E v e n t / Y^{k}\right)$ and no event: $\operatorname{Pr}\left(\right.$ No_event $\left./ Y^{k}\right)$.

Figure 9 shows the performance of GMM on a raw temperature dataset generated by the PIR sensors. As can be seen from this Figure, the GMM algorithm allows a reliable and accurate detection of vehicle events, as illustrated in Table II.

Table II. FALSE POSITIVES AND MISS DETECTION RESULTS OF GMM ALGORITHM.

\begin{tabular}{|l|l|l|l|}
\hline Sensor ID & Precision (\%) & Recall (\%) & Accuracy(\%) \\
\hline Sensor 1 & 97.78 & 96.70 & 99.98 \\
\hline Sensor 2 & 94.67 & 95.95 & 99.98 \\
\hline Sensor 3 & 92.80 & 99.15 & 99.97 \\
\hline
\end{tabular}

\section{Speed estimation}

Once the events have been identified by the GMM algorithm, the problem of estimating the vehicle length and speed boils down to an estimation of the delay occurring between the detection by consecutive sensors (each passive infrared sensors covers a given region of the road), and the total duration of the detection. The actual shape of the temperature signal can also be used to infer and classify the vehicle type.

The speed estimation algorithm in particular relies on a constrained cross correlation method in which we search for the maximal correlation occurring during a feasible time period. This time period is constrained by the average performance of the vehicles, and the speed limit of the road. It can be summarized as:

$$
\begin{array}{ll}
\underset{\tau}{\arg \max } & R_{x x(t, t+\tau)} \\
\text { subject to } & \tau_{\min } \succeq \tau_{i} \succeq \tau_{\max },
\end{array}
$$

To mitigate the effects of sensor noise, a robust maximization approach can instead be considered, particularly if

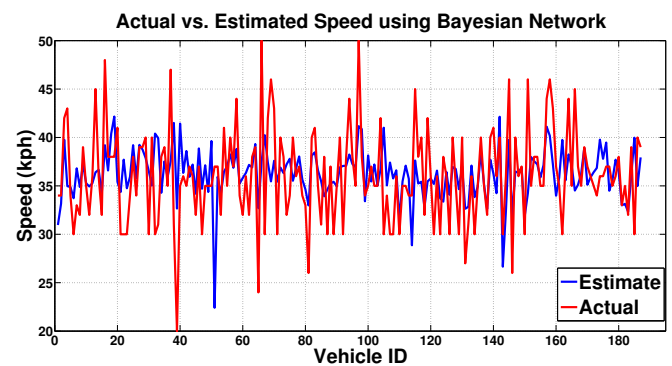

Figure 10. Actual vs. estimated vehicle speed based on Bayesian inference using PIR sensors and ultrasound measurements

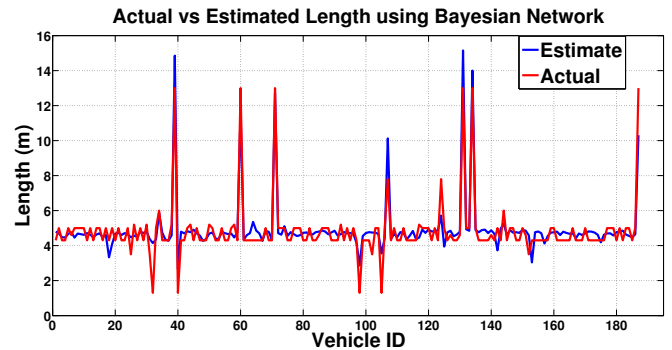

Figure 11. Actual vs. estimated vehicle length based on Bayesian inference using PIR sensors and ultrasound measurements

the temperature contrast between the vehicle and the ground (which drives the signal to noise ratio) is low. This occurs for instance in situations in which the wind is high and the temperatures are mild (no A/C nor heater, and relatively high heat transfer between the vehicle and the air). Estimated delay along with the duration of event are used to estimate the speed and the length of the passing vehicle using Bayesian inference. The performance of the sensor in terms of speed estimation and vehicle length estimation is shown in Figure 10 and Figure 11. The speed and vehicle length estimation is based on Bayesian inference, using the properties of the events detected by the GMM (in particular their durations and temporal delays, though other criteria could also be used).

As can be seen from this Figure, the error in speed estimation is acceptably low (mean error of $5 \mathrm{kph}$ in speed estimation), and the vehicle lengths are reliably estimated. Vehicle length is a very important metric for vehicle classification, which allows more accurate traffic sensing.

Once the speed and the length of the vehicle are estimated, we can use for instance ANN or Bayesian inference to classify the vehicle types. We have classified the vehicles in five different categories, namely: sedan vehicle, Pick up truck, offroad vehicle, bus, and two wheeler. The classification results are shown in the below table.

Table III. PERFormanCE METRICS OF VEHIClE ClASSIFICATION USING PIR AND ULTRASOUND SENSORS USING NN AND BN

\begin{tabular}{|l|l|c|c|c|l|}
\hline \multirow{2}{*}{ Class } & \multicolumn{2}{|c|}{ Recall (\%) } & \multicolumn{2}{c|}{ Precision (\%) } & \multirow{2}{*}{ Num. of Instances } \\
\cline { 2 - 5 } & BN & NN & BN & NN & \\
\hline Sedan vehicle & 94.50 & 92.04 & 99.04 & 86.67 & 109 \\
\hline Pick up truck & 86.96 & 47.83 & 100 & 84.62 & 23 \\
\hline off-road vehicle & 40 & 88.89 & 100 & 86.96 & 43 \\
\hline Bus & 100 & 90.00 & 100 & 90.00 & 8 \\
\hline two wheeler & 100 & 50 & 100 & 40.00 & 4 \\
\hline
\end{tabular}




\section{CONCLUSION AND FUTURE WORK}

In this article, we presented a new type of wireless sensor platform applicable to urban traffic flow and flash flood monitoring, as well as processing algorithms enabling one to monitor both phenomena simultaneously. The proposed sensor comprises an ultrasonic rangefinder and one or multiple passive infrared temperature sensors. Because of the extremely low absolute distance measurement error required by the system (on the order of $0.2 \%$ ), one needs to accurately estimate the temperature profile of the air layer between the sensor and the ground, since the perturbations of the speed of sound caused by temperature changes are significant. Since this profile is impossible to model accurately (because of unknown model parameters and unknown boundary conditions), we chose a non model-based approach for estimating the correction due to deviations in temperature. Our article shows that Artificial Neural Networks (ANNs) capture the effects of the underlying model very accurately, and can be used to monitor water level in streets in real time. We also show that the same sensor can accurately monitor traffic flow conditions. Since flash floods are very rare events, a sensor capable of monitoring flash floods only makes little economic sense, and multi-capability sensors are critical. Future work will be dedicated to the deployment of a larger number of flash flood/traffic sensor nodes, and their integration within traffic monitoring systems and flash flood monitoring systems, to assess the performance of the complete system (particularly for the flash flood sensing component).

\section{REFERENCES}

[1] ISDR disaster statistics, http://www.unisdr.org/.

[2] Turn around, don't drown (US National Weather Service), http://www.nws.noaa.gov/os/water/tadd/tadd-intro.shtml.

[3] L. Li, Y. Hong, J. Wang, R. Adler, F. Policelli, S. Habib, D. Irwin, T. Korme, and L. Okello. Evaluation of the real-time TRMM-based multi-satellite precipitation analysis for an operational flood prediction system in Nzoia Basin, Lake Victoria, Africa. Natural hazards, 50(1):109-123, 2009.

[4] S. A. Ahmed, T. Hussain, and T. N. Saadawi. Active and passive infrared sensors for vehicular traffic control. In Vehicular Technology Conference, 1994 IEEE 44th, pages 1393-1397. IEEE, 1994.

[5] G. Alessandretti, A. Broggi, and P. Cerri. Vehicle and guard rail detection using radar and vision data fusion. Intelligent Transportation Systems, IEEE Transactions on, 8(1):95-105, 2007.

[6] R. Alonso, M. Santillana, and C. Dawson. On the diffusive wave approximation of the shallow water equations. European Journal of Applied Mathematics, 19(05):575-606, 2008.

[7] E. A. Basha, S. Ravela, and D. Rus. Model-based monitoring for early warning flood detection. In Proceedings of the 6th ACM conference on Embedded network sensor systems, pages 295-308. ACM, 2008.

[8] S. Ben-Yaakov, C. Golan, and S. Kesler. A stand-alone ultrasonic water stage monitoring system. In Industrial Electronics, 1992., Proceedings of the IEEE International Symposium on, pages 437-441. IEEE, 1992.

[9] M. Borga, E. Anagnostou, G. Blöschl, and J.-D. Creutin. Flash flood forecasting, warning and risk management: the hydrate project. Environmental Science \& Policy, 14(7):834-844, 2011.

[10] M. Bramberger, J. Brunner, B. Rinner, and H. Schwabach. Real-time video analysis on an embedded smart camera for traffic surveillance. In Real-Time and Embedded Technology and Applications Symposium, 2004. Proceedings. RTAS 2004. 10th IEEE, pages 174-181. IEEE, 2004.

[11] R. H. Brown. Liquid Level Sensor, Dec. 16 1997. US Patent 5,697,248.
[12] S.-Y. Cheung and P. P. Varaiya. Traffic surveillance by wireless sensor networks: Final report. 2007.

[13] C. M. Day, H. Premachandra, T. M. Brennan, J. R. Sturdevant, and D. M. Bullock. Operational evaluation of wireless magnetometer vehicle detectors at signalized intersection. Transportation Research Record: Journal of the Transportation Research Board, 2192(1):11-23, 2010.

[14] A. H. Dehwah, M. Mousa, and C. G. Claudel. Lessons learned on solar powered wireless sensor network deployments in urban, desert environments. Ad Hoc Networks, 28:52-67, 2015.

[15] K. Derr and M. Manic. Wireless based object tracking based on neural networks. In Industrial Electronics and Applications, 2008. ICIEA 2008. 3rd IEEE Conference on, pages 308-313. IEEE, 2008.

[16] K. B. Eom. Analysis of acoustic signatures from moving vehicles using time-varying autoregressive models. Multidimensional Systems and Signal Processing, 10(4):357-378, 1999.

[17] S. Erb. Classification of vehicles based on acoustic features. na, 2007.

[18] R. Fante. Turbulence-induced distortion of synthetic aperture radar images. Geoscience and Remote Sensing, IEEE Transactions on, 32(4):958 -961, jul 1994.

[19] V. C. Gungor and G. P. Hancke. Industrial wireless sensor networks: Challenges, design principles, and technical approaches. Industrial Electronics, IEEE Transactions on, 56(10):4258-4265, 2009.

[20] K. Hornik, M. Stinchcombe, and H. White. Multilayer feedforward networks are universal approximators. Neural networks, 2(5):359-366, 1989.

[21] Z. Jia, C. Chen, B. Coifman, and P. Varaiya. The PEMS algorithms for accurate, real-time estimates of g-factors and speeds from single-loop detectors. In Intelligent Transportation Systems, 2001. Proceedings. 2001 IEEE, pages 536-541. IEEE, 2001.

[22] J. Jiang and C. Claudel. A wireless computational platform for distributed computing based traffic monitoring involving mixed eulerianlagrangian sensing. In Industrial Embedded Systems (SIES), 2018 8th IEEE International Symposium on, pages 232-239. IEEE, 2013.

[23] Y. Jo and I. Jung. Analysis of vehicle detection with WSN-based ultrasonic sensors. Sensors, 14(8):14050-14069, 2014.

[24] S. N. Jonkman and I. Kelman. An analysis of the causes and circumstances of flood disaster deaths. Disasters, 29(1):75-97, 2005.

[25] Y.-K. Ki and D.-K. Baik. Vehicle-classification algorithm for singleloop detectors using neural networks. Vehicular Technology, IEEE Transactions on, 55(6):1704-1711, 2006.

[26] M. K. Lindell and C. S. Prater. Assessing community impacts of natural disasters. Natural Hazards Review, 4(4):176-185, 2003.

[27] P. Milly, R. Wetherald, K. Dunne, and T. Delworth. Increasing risk of great floods in a changing climate. Nature, 415(6871):514-517, 2002.

[28] M. Papageorgiou. Applications of automatic control concepts to traffic flow modeling and control. 1983.

[29] J. V. Phillips and S. Tadayon. Selection of Manning's roughness coefficient for natural and constructed vegetated and non-vegetated channels, and vegetation maintenance plan guidelines for vegetated channels in Central Arizona. Citeseer, 2006.

[30] V. Sakharov, S. Kuznetsov, B. Zaitsev, I. Kuznetsova, and S. Joshi. Liquid Level Sensor Using Ultrasonic Lamb Waves. Ultrasonics, 41(4):319-322, 2003.

[31] T. N. Schoepflin and D. J. Dailey. Algorithms for calibrating roadside traffic cameras and estimating mean vehicle speed. In Intelligent Transportation Systems Conference, 2007. ITSC 2007. IEEE, pages 277-283. IEEE, 2007.

[32] H. G. Sohn, J. Heo, H. Yoo, S. Kim, and H.Cho. Hierarchical multi sensor approach for the assessment of flood related damages. Proceedings of the 27th ISPRS congress, 2008.

[33] I. Sraj, K. T. Mandli, O. M. Knio, C. N. Dawson, and I. Hoteit. Uncertainty quantification and inference of manning's friction coefficients using DART buoy data during the tōhoku tsunami. Ocean Modelling, 83:82-97, 2014. 
[34] O. P. Tossavainen, J. Percelay, A. Tinka, Q.Wu, and A. Bayen. Ensemble Kalman Filter based state estimation in $2 \mathrm{~d}$ shallow water equations using Lagrangian sensing and state augmentation. In 47th IEEE Conference on Decision and Control, 2008, pages 1783-1790. IEEE, 2008.

[35] N. S. USHIO. Loop vs ultrasonic in chicago: Ultrasonic vehicle detector field test isolating diffused reflection and enduring harsh environment. In Towards the new horizon Together. Proceedings of the 5th world congress on intelligent transport systems, held 12-16 October 1998, Seoul, Korea. paper No. 3048, 1998.

[36] P. Zappi, E. Farella, and L. Benini. Tracking motion direction and distance with pyroelectric IR sensors. Sensors Journal, IEEE, 10(9):14861494, 2010.

[37] W. Zhang, G. Tan, H.-M. Shi, and M.-W. Lin. A distributed threshold algorithm for vehicle classification based on binary proximity sensors and intelligent neuron classifier. J. Inf. Sci. Eng., 26(3):769-783, 2010. 